\title{
Review
}

\section{Human benign prostatic hyperplasia heterotransplants as an experimental model}

\author{
Lluis-A Lopez-Barcons \\ Stanley S Scott Cancer Center, Louisiana State University, Health Sciences Center, New Orleans, LA 70112, USA
}

\begin{abstract}
Benign prostatic hyperplasia is a nonmalignant adenomatous enlargement of the periurethral prostate gland. It is a common disease in older men. In addition to man, spontaneous benign prostatic hyperplasia occurs in chimpanzee and the dog. Alternatives to these spontaneous models are induced benign prostatic hyperplasia, xenografts and in vitro models. Xenografts may be induced by cells cultured in vitro or by the heterotransplantation of primary surgical specimens into immunosuppressed mice. The purpose of this review is to integrate data from more than 30 years of heterotransplantation research in the study of benign hyperplasia of the prostate. Heterotransplantation has provided data regarding the histopathology, morphology, tissue markers, androgen receptor expression, tissue kinetics, take rate and tissue vasculature for this prostate disease. There are advantages, as well as limitations, that have been identified for human prostate disease heterotransplants versus xenotransplantation of cultured cells. Overall, heterotransplanted tissue is better at retaining tissue morphology, pathology, secretory activity, expression of tissue markers and human vasculature of the patient's original specimen. Furthermore, heterotransplanted tissue preserves the three-dimensional tissular architecture of the prostate to maintain critical stromal-epithelial cell interactions.
\end{abstract}

Asian Journal of Andrology (2010)12: 157-163. doi: 10.1038/aja.2009.77; published online 30 November 2009.

Keywords: benign prostatic hyperplasia, heterotransplant, nude mice, prostate, xenotransplant

\section{Introduction}

Benign prostatic hyperplasia $(\mathrm{BPH})$ is a nonmalignant enlargement of the prostate due to cellular hyperplasia (increased cellular number) and hypertrophy (increased cellular size) [1]. These cellular parameters can affect both the epithelial and the stromal elements of the prostate gland, with the cell type affected varying greatly between patients [1]. Light and electron stereological analysis of

Correspondence to: Dr Lluis-A Lopez-Barcons, Stanley S Scott Cancer Center, Louisiana State University, Health Sciences Center, New Orleans, LA 70112, USA.

Fax:+1-504-568-6888_E-mail: lalb93@gmail.com

Received: 7 August 2009 Revised: 14 September 2009

Accepted: 10 November 2009 Published online: 30 November 2009 normal human prostate compared with BPH has shown that $\mathrm{BPH}$ is predominantly a stromal (fibromuscular) disease [1]. Direct interactions between the underlying fibromuscular stroma and the overlying epithelium seem to be essential to both the morphogenesis and the maintenance of the prostate. BPH is a disease that develops spontaneously in men and progresses slowly. Autopsy studies have repeatedly shown an association between BPH and aging based on histological criteria, prostate weight and prostate volume [1]. The disease is marked by urethral obstruction caused by prostate enlargement that can progress to acute urinary retention. Treatment of BPH includes the use of $\alpha$-blockers to relax smooth muscles present in the prostate stroma, administration of $5 \alpha$-reductase inhibitors to reduce formation of $5 \alpha$-dihydrotestosterone (DHT), which 
is responsible for normal prostate maintenance and homeostasis, and finally, surgery to relieve severe urethral obstruction. BPH is a common health problem in the United States. In 2000, 4.5 million patients consulted their physicians with symptoms of BPH [2]. Although BPH rarely causes symptoms before the age of 40 years, more than $50 \%$ of men in their sixties, and as many as $90 \%$ in their seventies and eighties, have symptoms [2]. It is estimated that by 2020,65 million Americans who are 65 years or older will be diagnosed with BPH [3]. Therefore, the availability of a relevant in vivo model to investigate the biology and potential treatments of prostate diseases is necessary.

In the field of preclinical experimental therapy, there is a great need for tumor models relevant to human prostate diseases. The use of mouse xenograft models to represent preclinical benign and malignant tumors has become more widespread as this model is reasonably inexpensive, can provide rapid experimental data, is largely free of regulatory constraints and can be used with many different specimen types. Artificially cultured human BPH epithelial cells, such as BPH-1, BPH-2 and BPH-3 [4], can be implanted in immunosuppressed mice. There have been an increasing number of studies using this approach. Despite the increased use of these xenograft models, there are disadvantages to this approach. First, not all cell subpopulations consistently maintain the genetic aberrations that enable the hyperplasic cells to be maintained in cell culture. Second, in vitro culturing over several years has the potential to select for certain cell subtypes that may or may be not relevant to $\mathrm{BPH}$, and therefore may no longer be representative of the original disease [5]. Third, the generation of cell lines for in vitro culturing currently includes only a limited number of benign biopsies. Taken together, these aspects of in vitro culturing suggest that the resulting subsets of prostate cells generated may not accurately represent the genetic diversity of the disease.

The prostate is a complex organ system with a distinct heterogeneous cell population that composes the epithelium. The cell types contained in the prostate include luminal and basal epithelial cells, neuroendocrine cells, smooth muscle cells, fibroblasts, nerves and basement membranes that interact to coordinate function in the prostate. Prostate stromal cells have been shown to have an important role in modulating the morphology and migration of prostate epithelial cells [6].
It is important to note that the necessary interactions between cell types of the prostate are not completely reconstituted in xenografts, as proteins from one species cannot always interact with their counterparts in the host. However, when co-implantation of human epithelial cells with human stromal cells is performed, the combination generally recapitulates the human disease. This approach has been taken by combining BPH-1 cells with prostate stromal cells in an elegant work performed by Barclay et al. [7]. However, the mixture with stromal cells obtained from normal prostate did not produce visible xenografts. On the other hand, when stromal cells from BPH were used, small, well-organized and sharply demarcated xenografts were obtained. However, one limitation of this approach is the use of rat-tail collagen as a supportive matrix for the cell mixture and its implantation in the renal capsule, as no subcutaneous (sc) implantation has been reported [7]. BPH models are further limited by the fact that in addition to humans, only primates and dogs are known to suffer from BPH with age $[8,9]$. Unfortunately, both of these animal models are very expensive to maintain and are subject to stringent regulatory constraints. Another possible BPH model involves rodents; however, the extrapolation of the results obtained with this model to human disease is limited by its nonhuman origin [10]. Alternatively, the prostate heterotransplant model includes all the cell types present during the development of $\mathrm{BPH}$, and the architecture of the gland is preserved. The spatial relationship between the stromal and the epithelial cell compartments is also maintained, along with the necessary vasculature, as the human cells are able to proliferate and differentiate [11].

To evaluate the $\mathrm{BPH}$ heterotransplant model, it is necessary to know how well the heterotransplanted tissue can maintain the anatomical architecture and function of the original hyperplasia collected from the patient. A key aspect of the heterotransplant model is that the human biopsy is inserted in a living host from a different species, and it is the interactions between these two microenvironments that have been studied for the past 30 years. It is in this body of work that human prostate tissue heterotransplantation has defined how human tissues maintain their integrity, and yet change as a result of their interactions with the host cells. The interactions that take place at this human-mouse interface of the heterotransplanted model are the focus of this review. 


\section{Materials and methods}

The published data from PubMed, http://www. ncbi.nlm.nih.gov, and Current Contents Connect, http:// thomsonreuters.com/.../scientific/Current_Content_ Connect, have been reviewed. In addition, references cited in each bibliographic reference have been used as another bibliographic source.

\section{Human BPH heterotransplants in immunosup- pressed mice. Similarities with the patient specimen of origin}

In the text, the standard heterotransplant implantation site for the patient's tissue is considered to be the sc space of the host mice. When other implantation sites are used, such as the subrenal capsule or the prostate itself, the location will be specifically cited.

\subsection{Cytological and histological analysis}

After the initial heterotransplantation of a patient's BPH specimen into a murine host, typically, the implanted BPH is viable and its morphology is similar or nearly identical to the original specimen. The human prostate origin of the heterotransplants is confirmed by in situ hybridization studies using DNA probes containing repetitive sequences of human DNA to identify epithelial cells and stromal fibroblasts $[12,13]$. Prostate glandular architecture has been found to be well preserved, as characterized by the stroma-epithelium interface that is maintained [11, 13-18]. In other studies, well-vascularized ducts and high columnar epithelium with secretory activity were found in proximity to acini-filled epithelial cells [19]. In early studies, heterotransplanted tissue was shown not to become anaplastic or atrophic, and the basic pathology remained unchanged even after serial passaging of the heterotransplants [13, 14]. Many stromal myofibroblasts and vascular endothelial cells (EC) exhibited strong staining for proliferating cell nuclear antigen (PCNA), indicating high proliferative activities in the glands and in the supporting mesenchymal cells. The proliferating epithelial and stromal cells were all of human origin without an evidence of host mesenchymal elements invading or replacing the interglandular spaces [13]. Remarkably, the histology of the BPH specimens was preserved in the heterotransplants, even when the patient tissue was cryopreserved before transplantation
[11]. However, there have been some cases in which minor histological differences between the original tissue and the heterotransplant have been reported. Incidents of stromal tissue inflammation, squamous or transitional epithelial cell metaplasia, basal cell hyperplasia and glandular atrophy have been described $[13,18,20]$. Similar results were reported when the patient tissue was implanted into the subrenal capsule [21]. In addition, minor histological differences have been recorded in $50 \%$ and $67 \%$ of heterotransplanted fragments 3 and 4 months after subcutaneous implantation, respectively [17].

The development of a heterotransplant model has included testing of various variables of the model. For example, the histology of patient BPH samples has been shown to be consistently independent of the host strain used. The mouse strains evaluated included CBA thymectomized and irradiated mice, athymic balb/c, Beige, KSN, NMRI, CD-1 nude mice and CB17 severely combined immunodeficient (SCID) mice. The use of Matrigel (Becton-Dickinson, Franklin Lakes, NJ, USA) has been another variable of the heterotransplant model. Matrigel (BectonDickinson) is an extracellular matrix gel composed of type-IV procollagen, laminin and heparan sulfate proteoglycan obtained from the Engelbreth-HolmSchwarm mouse sarcoma first described by Kleinman et al. [22]. The matrix has been implanted with human $\mathrm{BPH}$ specimens to increase the heterotransplant take rate. Additional variables of the heterotransplant model include the site of implantation and androgen supplementation. Wang et al. [23] reported the heterotransplantation of BHP ectopically into the subrenal capsule, the sc space and orthotopically in the prostate of orchiectomized scid mice supplemented with testosterone (T) pellets. They found that the implantation site influenced the response of the heterotransplanted tissue, and gland inflammation was rarely observed at any of the sites. However, after 3 months incubation, the ratio of gland tissue to stroma was clearly higher at the orthotopic site compared with the subcapsular and sc sites. Stromal inflammation was found to be very mild at the orthotopic site, and was mild to moderate in the subcapsular and sc space. In addition, basal cell hyperplasia was mild at the orthotopic and sc sites, and minimal at the renal subcapsular site. Finally, atrophic changes were mild to moderate at the orthotopic and subcapsular sites, and moderate to severe in the sc space. 


\subsection{Marker expression and functional activity}

When BPH tissue is heterotransplanted, active secretory luminal epithelial cells can be identified that positively stain for cytoplasmic prostate acid phosphatase (PAP) and prostate serum antigen (PSA) [12, 14, 17, 19]. Similar results have been obtained using patient specimens that were implanted in the subrenal capsule [23]. These heterotransplants either positively stained for PSA or PAP, and in each case, the stainings were consistent with the staining of the original patient specimens $[21,23]$. After 3 weeks, orchiectomy of the host mice revealed that the PSA staining was undetectable in most epithelial formations and was detected at low levels in only a few residual patches [21]. As in humans, the serum PSA mean value for all BPH heterotransplant-bearing mice was high $(19.03 \pm 13.36$ ng $\mathrm{mL}^{-1}$ ) compared with control mice serum PSA values $\left(1.59 \pm 1.66 \mathrm{ng} \mathrm{mL}^{-1}\right)$ [15].

\subsection{Hormone dependence}

In 1978, Williams et al. [14] administered titrated T to $\mathrm{BPH}$ heterotransplant-bearing mice and showed that the heterotransplanted BPH tissue maintained its ability to take up and concentrate $\mathrm{T}$ at two to three times the rate of control heterotransplanted tissue. Since then, additional studies have shown that heterotransplanted hyperplastic tissue in host mice is influenced by androgens. For example, exponentially growing heterotransplants in host mice were found to regress after bilateral orchiectomy of the host. These results are consistent with the tissue flattening and metaplastic changes previously described in BPH patients who underwent surgical or medical castration [23]. Staack et al. [21] stained the first heterotransplanted $\mathrm{BPH}$ tissue for androgen receptor (AR) expression and ARs were found uniformly expressed in most epithelial cells and in many stromal cells. However, 3 weeks after host orchiectomy, AR expression became patchy and was primarily identified in only a few epithelial cells. Furthermore, the staining of the epithelial cells was also markedly less intense in the stromal compartment. Androgen deprivation caused by orchiectomy resulted in the regression of the benign hyperplastic tumor volume. At the molecular level, the apoptotic index (AP) was determined from orchiectomized hosts relative to intact hosts. The highest AP values were observed on days 4, 7 and 8, which were 52-, 30- and 30-times higher than the baseline value, respectively. Eventually, the AP values gradually decreased until day 21 . These results correspond to an overall loss of $87 \%$ of the total hyperplastic epithelial cell population, indicating that only $13 \%$ of the epithelial cells survived the host orchiectomy.

Hormonal supplementation of orchiectomized mice with T or with a combination of DHT and 17- $\beta$-estradiol (E) before heterotransplantation of the $\mathrm{BPH}$ tissues resulted in the growth of the implanted fragments. In the presence of hormone supplementation, dilated glandular structures were observed that produced a cystic pattern with wide epidermoid foci, and also the proliferation of luminal secretory and basal epithelial cells similar to the original patient tissue was determined in situ $[16,19]$. Hormonal supplementation was also associated with the functional preservation of the epithelium, as characterized by the presence of numerous secretory vacuoles, few dense bodies, rough endoplasmic reticulum, Golgi apparatus and clear cellular outlines [19]. In contrast, hormonally deprived mice receiving heterotransplanted tissue had a narrow lumen, whereas squamous epithelial metaplasia occurred when androgens were lacking or when estrogens were applied systemically. To limit the extent of tissue metaplasia, $\mathrm{T}$ pellet implants were used. A reduction in tissular squamous metaplasia was observed and correlated with the functional preservation of the heterotransplanted tissue. In BPH heterotransplants, nuclei of epithelial, connective cells, basal cell hyperplasias and some cystic epithelium positively stained for AR expression. In contrast, most of the squamous-cell metaplasias were negative for AR expression.

\subsection{Frequency of mitosis}

A quantitative analysis of the original patient $\mathrm{BPH}$ specimen and the corresponding heterotransplant, 1 month after implantation, was performed. The proliferating index, or number of proliferating cells, was determined by staining cells for expression of Ki67 (a cell proliferation-associated nuclear antigen). There were no remarkable differences in $40 \%$ of the $\mathrm{BPH}$ tissues studied (two of five) or in the AP index analysis in $80 \%$ of the cases studied (four of five) [11]. Cell proliferation was also evaluated using staining for PCNA in epithelial and stromal cells of the BPH heterotransplants in host mice fed with DHT and hydrocortisone versus control mice. The proliferation index of the prostate tissues from mice fed with DHT and hydrocortisone was not different from that of the prostate tissues from nontreated mice [12, 13, 17]. Different implantation sites were also tested and 
no differences were found between implantation sites in the renal subcapsule, the peritoneal pouch or in the sc space $[12,13]$.

\subsection{Heterotransplantation take rate}

Currently, histological examinations are used to evaluate whether a human specimen heterotransplantation is successful. If viable BPH tissue can be detected after implantation of the specimen in the host, the heterotransplantation is considered to have occurred. For a realistic approach, the successful number of heterotransplanted mice from one single patient is considered as one success only, independent of the number of mice heterotransplanted with the same specimen obtained from a given patient in the first heterotransplantation. The initial take rate for prostate hyperplastic tumors heterotransplanted into mice is very low, with $3 \%$ (three of 66) and $0 \%$ (0 of nine) of heterotransplants successfully achieved using athymic nude mice with or without administration of $\mathrm{T}$ pellets, respectively [24]. However, when the host mice are from the NMRI nude mice strain, with and without DHT plus E supplementation, the take rate improves to $100 \%$ (two of two; three of three) for both treatment groups $[16,19]$. Interestingly, similar results were obtained when hyperplastic tissue was heterotransplanted in combination with Matrigel (Becton-Dickinson) using athymic nude mice supplemented with $\mathrm{T}$ pellets. The take rates were $100 \%$ (eight of eight) and $97.3 \%$ (41 of 42) with or without administration of DHT+E, respectively $[11,18]$. In contrast, take rates of $100 \%$ and $58.1 \%$ were obtained when scid mice received $\mathrm{T}$ pellets and implants were made with and without Matrigel (Becton-Dickinson), respectively [15, 23]. Furthermore, when scid mice receiving $\mathrm{T}$ pellets were orchiectomized, the take rate was $93.4 \%$ (114 of 122 ) and $71.9 \%$ (41 of 57) for implantation sites in the subrenal capsule and prostate orthotopic site, respectively [23].

\subsection{Vasculature}

In studies by Gray et al. [18] with heterotransplanted tissues combined with Matrigel (Becton-Dickinson), tissues did not show areas of necrosis 1 month after implantation. These data show that the efficiency of angiogenesis and the rapidity of perfusion associated with the growing BPH tumor were sufficient to ensure viability of the entire heterotransplant. The average number of vessels per field, or mean vessel density (MVD), measured in high vascular fields was remarkably increased in the heterotransplanted tissue compared with the original patient's benign tumor by 4.8 -fold, 2.6 -fold and 6.9 -fold when the tissue was stained with the human anti-CD31 (PECAM-1) antibody, the human anti-CD34 (myeloid progenitor cell antigen) antibody and the human anti-VWF (factor VIII-related antigens) antibody, respectively. Correspondingly, the average number of vessels with at least one proliferating EC per field (that is, proliferative MVD) increased by eight-fold in the heterotransplanted tissue compared with in situ studies of the original patient benign tumor. Proliferating ECs were observed near the glandular structures and in vessels dispersed within the stroma. Although the mean vessel area of the heterotransplanted tissue was reduced by seven-fold compared with the original patient specimen, the mean vessel perimeter was reduced by 2.5 -fold. The five-fold increase in MVD was based on an increased number of vessels that were small to medium in diameter. As suggested by Gray et al. [18], an increase in the number of small vessels in the heterotransplant is consistent with the typical definition of angiogenesis, wherein smaller vessels grow from existing ones based on EC proliferation. The percentage of human vessels observed in the first heterotransplant was $61.0 \% \pm 13.4 \%$. The human origin of the capillary ECs observed in the heterotransplanted BPH fragments was confirmed by in situ hybridization using a DNA probe of human repetitive sequences $[12,13]$. In contrast, the blood vessels that stained with an anti-mouse-specific antibody mainly localized to a compressed connective tissue layer at the periphery of the heterotransplanted tissue. It is hypothesized that the mouse cells were derived from the connective tissue of the host and that some murine vessels can penetrate the heterotransplants as part of the angiogenesis generated by the host vasculature. In fact, with a longer implantation time, human prostate heterotransplants undergo an increased infiltration of mouse blood vessels. The gradual substitution of the human vasculature of the heterotransplant with murine vessels is hypothesized to be the result of a serial dilution of human angiogenic signaling molecules that occurs when the heterotransplants are serially harvested, fragmented and re-implanted. Consequently, the human vasculature present in the human prostate heterotransplant can be substituted with murine-derived vasculature with longterm passaging of heterotransplants [18]. 


\section{Experimental limitations in the use of human hetero- transplants}

Access to, and availability of, human specimens requires a coordinated team of highly trained clinical professionals including oncologists, surgeons, pathologists, internists, nurses, social workers and paralegals. In addition, these professionals have to coordinate their efforts with a team of researchers and laboratory assistants who maintain a database of samples and data to ensure the proper storage, analysis and implantation of patient specimens into immunosuppressed mice. Communication between these two groups is one of the challenges in performing human heterotransplant experiments.

A second challenge for human prostate heterotransplant experiments is the inevitable contamination of samples by host cells. For example, during the serial passaging of primarily stromal cells, host vasculature and infiltration of host inflammatory cells are present and are not able to be easily separated. Therefore, in situ hybridization using human probes and immunocytochemistry using antibodies against human epitopes help identify human versus mouse tissue in heterotransplants in spite of highly conserved sequences shared between human and mouse homologs [25].

\section{Experimental advantages of human heterotransplant models}

When patient specimens are initially heterotransplanted into the host mice, a majority of them maintain the biology of the original patient tissue. For example, the heterotransplanted tissue does not become anaplastic and the basic pathology remains unchanged even after serial passaging. Histology of the prostate gland is also well preserved with epithelial cells lining the gland adjacent to the stroma. Preservation of stromal cellepithelial cell interactions from the original primary $\mathrm{BPH}$ is important. Only in a few cases has mild stromal tissue inflammation, mild epithelial cell metaplasia or moderate glandular atrophy been reported.

Regarding $\mathrm{BPH}$, the heterotransplanted tissue, similar to the original patient tissue, has active secretory epithelial cells that express PSA and PAP. However, heterotransplants have been shown to lose AR expression that was originally detected in the patient specimens before implantation. In terms of cell cycle distribution, even after serial transplantation, the heterotransplanted hyperplasia cells have a similar cell cycle distribution as that of original patient specimens. Furthermore, the proliferation and apoptosis indices determined from the patient specimens versus the heterotransplanted tissue are similar. Remarkably, the heterotransplants retain a high percentage of human vessels in their vasculature. Taken together, these data indicate that the heterotransplant model is largely representative of BPH disease.

The human BPH heterotransplant model provides an opportunity to study the expression of human genes within a primarily in vivo human context and within the particular human prostate tissular environment that strongly influences gene expression and cell growth. The behavior of the cells can be studied, especially in response to experimental therapies. Proteomic and pharmacogenomic data can also be collected. As the heterotransplant model facilitates the growth of human BPH, new drugs, specially type-II $5 \alpha$-reductase inhibitors and aromatase inhibitors alone or in combination, can be evaluated for preventing progression of growth or even shrink the enlarged prostate heterotransplant. In addition, new therapeutic regimens for the treatment of the disease can be tested and the response of human vasculature to novel antiangiogenic therapies can be investigated. In all of these applications, the ability to cryopreserve a part of the patient specimens before heterotransplantation evidently increases the flexibility of the heterotransplant model.

\section{Conclusion}

The majority of heterotransplanted tissues in host mice have been shown to retain the biological properties that characterize the original human primary specimen collected. These properties include tissue morphology, pathology, secretory activity and the expression of important BPH tissue markers. Preservation of the original three-dimensional prostate architecture is significant for maintaining stromal cellepithelial cell interactions and the needed vasculature. Heterotransplantation of the human prostate specimens provides a model that is qualitatively superior to a xenograft model that uses cells cultured in vitro and results in a hyperplasia dominated by a murine-based stromal and vascular environment. The heterotransplant model has been shown to provide long-term growth for a variety of human BPHs, which facilitates the 
investigation of new agents and therapeutic protocols for its treatment. This model can also be applied to the study of angiogenesis in the benign human hyperplasia or for the evaluation of new anti-angiogenic therapies. Furthermore, the human heterotransplant model has the potential to provide proteomic and pharmacogenomic data relevant to the in vivo situation. In summary, implantation of human benign hyperplasia specimens into a mouse host provides a long-term, propagatable in vivo heterotransplant model of $\mathrm{BPH}$, and represents a significant advance in the ability to develop experimental therapies for this prostate disease that may be translated into clinical trials in future.

\section{References}

1 Stern JA, Fitzpatrick JM, McVary KT. Prostate anatomy and causative theories pathophysiology, and natural history of benign prostatic hyperplasia. In: McVary KT, editor. Management of benign prostatic hypertrophy. Totowa, New Jersey: Humana Press Inc; 2004. p1-20.

2 National Institutes of Diabetes and Digestive and Kidney Diseases (NIDDK). USA: NIH Publications; 2006. No. 07-3012.

3 Holtgrewe HL. Economic issues and the management of benign prostatic hyperplasia. Urology 1995; 3 (Suppl A): 23-5.

4 Krishnan AV, Peehl DM, Feldman D. Vitamin D and Prostate cancer. In: Feldman D, Glorieux FH, Pike JW, editors. Vitamin D, 2nd edn. Burlington, Massachusetts: Elsevier Academic Press; 2005. p1679-708.

5 Habib FK, Ross M, Bayne CW, Grigor K, Buck AC, et al. The localisation and expression of $5 \alpha$-reductase types I and II mRNAs in human hyperplastic prostate and in prostate primary cultures. J Endocrinol 1998; 156: 509-17.

6 Hall JA, Maitland NJ, Stower M, Lang SH. Primary prostate stromal cells modulate the morphology and migration of primary prostate epithelial cells in type 1 collagen gels. Cancer Res 2002; 62: 58-62.

7 Barclay WW, Woodruff RD, Hall MC, Cramer SD. A system for studying epithelial-stromal interactions reveals distinct inductive abilities of stromal cells from benign prostatic hyperplasia and prostate cancer. Endocrinol 2005; 146: 13-8.

8 De Marzo AM, Coffey DS, Nelson WG. New concepts in tissue specificity for prostate cancer and benign prostatic hyperplasia. Urology 1999; 53: 29-39.

9 Ferreira BR, Bechara GH, Pissinatti A, Cruz JB. Benign prostatic hyperplasia in the nonhuman primate Leontopithecus. Folia Primatol 1995; 65: 48-53.

10 Shappell SB, Thomas GV, Roberts RL, Herbert R, Ittmann $\mathrm{MM}$, et al. Prostate pathology of genetically engineered mice: definitions and classification. The consensus report from the Bar Harbor meeting of the Mouse Models Of Human Cancer Consortium Prostate Pathology Committee. Cancer Res 2004; 64: 2270-5.
11 Presnell SC, Werdin ES, Maygarden S, Mohler JL, Smith GJ. Establishment of short-term primary human prostate xenografts for the study of prostate biology and cancer. Am J Pathol 2001; 159: 855-60.

12 Debiec-Richter M, Asgari K, Jones RF, Grignon DJ, Haas GP, et al. Maintenance of human benign prostatic hyperplasia tissues in the nude mouse. Anticancer Res 1995; 14: 943-6.

13 Soos G, Debiec-Richter M, Jones RF, Zukowki K, Haas GP, et al. Maintenance of human hyperplastic prostate implants at different sites in athymic mice. Anticancer Res 1995; 15 : 2007-12.

14 Williams G, Ghanadian R, Castro JE. The growth and viability of human prostatic tissue maintained in immunosuppressed mice. Clin Oncol 1978; 4: 347-51.

15 Lubaroff DM, Cohen MB, Schultz LD, Beamer WG. Survival of human prostate carcinoma, benign hyperplastic prostate tissues, and IL-2-activated lymphocytes in SCID mice. Prostate 1995; 27: 32-41.

16 Otto U, Wagner B, Becker H, Schroeder S, Klosterhalfen $\mathrm{H}$. Transplantation of human benign hyperplastic prostate tissue into nude mice: first results of systemic therapy. Urol Int 1992; 48: 167-70.

17 Debiec-Richter M, Asgari K, Jones RF, Grignon DJ, Haas GP, et al. Xenografts of human benign prostatic hyperplasia tissues in the nude mouse. In Vivo 1994; 8: 449-60.

18 Gray DR, Huss WJ, Yau JM, Durham LE, Werdin ES, et al. Short-term human primary xenografts: an in vivo model of human prostate cancer vasculature and angiogenesis. Cancer Res 2004; 64: 1712-21.

19 Claus S, Aumuller G, Tunn S, Senge T, Schulze H. Influence of hormone application by subcutaneous injections or steroid-containing silastic implants on human benign hyperplastic prostate tissue transplanted into male nude mice. Prostate 1993; 22: 199-205.

20 Yamada Y, Tozawa K, Cui L, Takahashi S, Shirai T, et al. Characteristics of nonneoplastic human prostate tissue transplanted into nude mice. Prostate 1998; 37: 109-15.

21 Staack A, Kassis AP, Olshen A, Wang Y, Wu D, et al. Quantitation of apoptotic activity following castration in human prostatic tissue in vivo. Prostate 2003; 54: 212-9.

22 Kleinman HK, McGarvey ML, Liotta LA, Robey PG, Tryggvason $\mathrm{K}$, et al. Isolation and characterization of type IV procollagen, laminin, and heparan sulfate proteoglycan from the EHS sarcoma. Biochemistry 1982; 21: 6188-93.

23 Wang Y, Revelo MP, Sudilovsky D, Cao M, Chen WC, et al. Development and characterization of efficient xenograft models for benign and malignant human prostate tissue. Prostate 2005; 64: 149-59.

24 Reid LM, Minato N, Gresser I, Holland J, Kadish A, et al. Influence of anti/mouse interferon serum on the growth and metastasis of tumor cells persistently infected with virus and of human prostatic tumors in athymic nude mice. Proc Natl Acad Sci USA 1981; 78: 1171-5.

25 Yang J, Liu A, Dougherty C, Chen X, Guzman R, et al. Beware of contaminating mouse cells in human xenografts from nude mice. Anticancer Res 2000; 20: 1635-40. 